\title{
Hypertension, chronic kidney disease, atrial fibrillation and the newer anticoagulants
}

\section{B Rayner MBChB, FCP, MMed}

To cite this article: B Rayner MBChB, FCP, MMed (2012) Hypertension, chronic kidney disease, atrial fibrillation and the newer anticoagulants, South African Family Practice, 54:5, 418-419, DOI: $10.1080 / 20786204.2012 .10874263$

To link to this article: http://dx.doi.org/10.1080/20786204.2012.10874263
○ (c) 2012 SAAFP. Published by Medpharm.

曲 Published online: 15 Aug 2014.

Submit your article to this journal $\pi$

山 Article views: 59

Q

View related articles ¿ 


\section{Hypertension, chronic kidney disease, atrial fibrillation and the newer anticoagulants}

Rayner B, MBChB, FCP, MMed Division of Nephrology and Hypertension, University of Cape Town Correspondence to: Brian Rayner, e-mail: brian.rayner@uct.ac.za Keywords: hypertension, chronic kidney disease, atrial fibrillation, anticoagulants

Atrial fibrillation (AF) is a common clinical condition that is associated with increased morbidity and mortality that mainly relates to an embolic stroke. Dominant risk factors for AF are advanced age and hypertension in the absence of mitral valve disease. ${ }^{1}$ In turn, hypertension and ageing are determinants of the congestive heart failure, hypertension, age, diabetes mellitus, prior stroke or transient ischaemic attack or thromboembolism $\left(\mathrm{CHADS}_{2}\right)$ criteria for assessing the indication for anticoagulation. In addition, they are important risk factors for chronic kidney disease (CKD). In itself, CKD is an independent risk factor for AF and a higher risk of stroke. ${ }^{2}$ It is highly likely that a practitioner will encounter older patients with $\mathrm{AF}$ and concomitant hypertension and CKD that require anticoagulation therapy. Thus, it is essential for the practitioner to understand the risks and benefits of anticoagulation in older patients with $\mathrm{AF}$, hypertension and CKD.

CKD is defined by glomerular filtration rate (GFR), usually estimated from the serum creatinine by a variety of equations, generally the Modification of Diet in Renal Disease in South Africa. CKD is defined by five stages. Stage 1: $90 \mathrm{ml} /$ minute, stage 2: $60-90 \mathrm{ml} /$ minute, stage $3: 30-60 \mathrm{ml} / \mathrm{min}$ ute, stage $4: 15-30 \mathrm{ml} /$ minute and stage 5 (or end-stage) $<15 \mathrm{ml} /$ minute. It is also important to recognise that the calculation of estimated GFR (eGFR) is an epidemiological tool that has useful clinical utility. However, there may be an important underestimation or overestimation of true GFR in the individual patient. This can be particularly pertinent in older people, who are frail with reduced muscle bulk renal function that may be underestimated. Kidney function is an important determinant of the pharmacokinetics of a variety of drugs. This is particularly important in drugs with a narrow therapeutic and toxic range, such as anticoagulants.

Historically, warfarin has been the mainstay of anticoagulation in patients with AF. However, a large observational study has suggested that compared to placebo, warfarin is associated with increased risk of stroke, both ischaemic and haemorrhagic, in patients with advanced CKD, compared to no therapy, even when international normalised ratio (INR) levels are within the therapeutic range. ${ }^{3}$ Warfarin is considered to be contraindicated in patients with CKD stages 4 and 5 . It also blocks Vitamin $\mathrm{K}_{2}$, which may be important in the prevention of vascular calcification, highly prevalent in the CKD population. ${ }^{4}$

Recent publications of major studies that attested to the safety and efficacy of the newer anticoagulants, dabigatran, rivaroxaban, and apixaban, heralded a new era in anticoagulation for patients with AF. In particular, dabigatran $150 \mathrm{mg}$ was shown to be superior to warfarin, and was registered for use by the US Food and Drug Administration. ${ }^{5-7}$ However, in 2012, there was concern about excess bleeding in older patients who were switched from warfarin to dabigatran by the Haematology Society of Australia and New Zealand. ${ }^{8}$ In two months, approximately 7000 patients started on dabigatran treatment. Seventyeight cases of haemorrhage were reported, of which 12 were major. Two thirds occurred in patients over 80 years old, $58 \%$ with renal impairment and 50\% with reduced mass. A recent report from the US has suggested that the bleeding risk in this population may even be higher than in that taking warfarin. ${ }^{9}$ Of note, in the Randomized Evaluation of Long-term Anticoagulant Therapy (RELY) study, patients were younger, heavier and with better kidney function. ${ }^{5}$ The recommendation of a single dose, without being cognisant of renal function, seems to have been an error. A better understanding of the pharmacokinetics of dabigatran is helpful in understanding the dangers of anticoagulation.

Eighty percent of dabigatran is excreted unchanged in the urine, and the pharmacokinetics is highly influenced by renal function, with marked prolongation of the half-life. ${ }^{10}$ Currently, it is now considered to be contraindicated in CKD stages 4 and 5 . There should be a dose reduction in CKD stage 3 . On the other hand, rivoraxaban has a different pharmacokinetic profile. ${ }^{9}$ Only one third is excreted unchanged in the urine and two thirds is metabolised. Concentrations and half-life are less influenced by renal 
function. In the Rivoraxaban versus Warfarin in Nonvalvular Atrial Fibrillation (ROCKET) study, rivaroxaban was used in a dose of $20 \mathrm{mg}$ daily with eGFR $\geq 50 \mathrm{ml} /$ minute, but reduced to $15 \mathrm{mg}$ if the eGFR was $30-49 \mathrm{ml} /$ minute. This provided invaluable information about its safety when it is used in patients with reduced renal function. There was no difference in the primary efficacy outcome in patients with reduced, compared to normal, renal function, but there was significant reduction in fatal haemorrhage..$^{11}$ Currently, it is recommended that the dose of rivaroxaban in atrial fibrillation should be reduced in CKD stage 3 from $20 \mathrm{mg}$ to $15 \mathrm{mg}$ daily. It has not been tested in patients with CKD stage 4 and is contraindicated in CKD stage 5 .

In the Apixaban versus Warfarin in Patients with Atrial Fibrillation (ARISTOTLE) trial, apixaban or matching placebo was administered twice daily. Apixaban was given in 5-mg doses, but interestingly, 2.5-mg doses were used in a subset of patients with two or more of the following criteria: age of at least 80 years, body weight of no more than $60 \mathrm{~kg}$ and serum creatinine level $\geq 133 \mu \mathrm{mol} / \mathrm{l}$. Clearly the investigators had learnt from experience, and in this subset, the primary efficacy and safety outcomes mirrored the main study results. ${ }^{7}$

These issues raise pertinent questions about the responsible prescribing of anticoagulants. There are no completely safe anticoagulants. Increased risk of haemorrhage needs to be balanced by the benefits of the prevention of thrombosis and embolic stroke. In the case of atrial fibrillation, every patient needs to be assessed for anticoagulation using either the $\mathrm{CHADS}_{2}$ or $\mathrm{CHA}_{2} \mathrm{DS}_{2}$ VASc criteria to determine the indication for anticoagulation. A score $\geq 1$ indicates a need for anticoagulation. This needs to be balanced against the risk of haemorrhage. The development of the hypertension, abnormal renal or liver function, stroke history, bleeding history or predisposition, labile INR, elderly (age $>65$ ) and drugs and alcohol use concomitantly (HAS-BLED) criteria (see Table I) is a useful tool to assess the risk of haemorrhage. ${ }^{12,13}$ Age, uncontrolled hypertension and CKD are important components of these criteria.

Although the new anticoagulants represent a significant advance over warfarin, chiefly because of the stability of

Table I: HAS-BLED criteria for assessing haemorrhage (the higher the score, the greater the risk)

\begin{tabular}{|c|l|c|}
\hline H & Hypertension $(>160)$ & 1 \\
\hline A & Abnormal renal or liver function & 1 or 2 \\
\hline S & Stroke & 1 \\
\hline B & Bleeding & 1 \\
\hline L & Labile INR $(<60 \%$ TR $)$ & 1 \\
\hline E & Elderly $(>65)$ & 1 \\
\hline D & Drugs and alcohol concomitantly & 1 or 2 \\
\hline
\end{tabular}

INR: international normalised ratio, TR: therapeutic range anticoagulation and significantly fewer drug interactions, this does not mean that "one dose fits all" and that there is no need for monitoring. There is a significant need to develop tools to assess the degree of anticoagulation, particularly in the high-risk population described above. In the author's opinion, this will create a significant safety net to avoid haemorrhagic complications that are caused by excessive anticoagulation and increase physician confidence in the newer drugs.

Another critically important need is to develop reliable antidotes to reverse life-threatening bleeding. At least with warfarin, the prolonged INR can be readily reversed by Vitamin $\mathrm{K}$ and fresh frozen plasma, but reversal of the effects of the newer anticoagulants needs to be established.

In conclusion, the treatment of elderly patients with $\mathrm{AF}$, with CKD and hypertension presents many challenges. Warfarin and dabigatran are contraindicated in CKD stages 4 and 5. Rivaroxaban and apixaban probably have better safety profiles in CKD, but further studies are required in this population which is generally excluded from clinical trials, and yet constitutes a significant number of patients with a clinical indication for anticoagulants.

\section{References}

1. Hypertension and atrial fibrillation: diagnostic approach, prevention and treatment. Position paper of the Working Group 'Hypertension Arrhythmias and Thrombosis' of the European Society of Hypertension. J Hypertens. 2012;30(2):239-252.

2. Horio $\mathrm{T}$, Iwashima $\mathrm{Y}$, Kamide $\mathrm{K}$, et al. Chronic kidney disease as an independent risk factor for new-onset atrial fibrillation in hypertensive patients. J Hypertens. 2010;28(8):1738-1744.

3. Chan KE, Lazarus JM, Thadhani R, Hakim RM. Warfarin risk associates with increased risk of stroke in haemodialysis patients with atrial fibrillation. J Am Soc Nephrol. 2009;20(10):2223-2233.

4. Ketteler M, Rothe $\mathrm{H}$, Krüger T, et al. Mechanisms and treatment of extraosseous calcification in chronic kidney disease. Nat Rev Nephrol. 2011;7(9):509-516.

5. Connely SJ, Ezekowitz MD, Yusuf S, et al. Dabigatran versus warfarin in patients with atrial fibrillation. N Engl J Med. 2009;361(12):1139-1151

6. Patel MR, Mahaffey KW, Garg J, et al. Rivaroxaban versus warfarin in nonvalvular atrial fibrillation. N Engl J Med. 2011;365(10):883-891.

7. Granger $\mathrm{CB}$, Alexander JH, McMurray JJV, et al. Apixaban versus warfarin in patients with atrial fibrillation. N Engl J Med. 2011;365(11):981-992.

8. Harper $P$, Young L, Merriman E. Bleeding risk with dabigatran in the frail elderly (letter). N Engl J Med. 2012;366(9):864-866

9. US bleeding ADRs higher with dabigatran than warfarin. TheHeart.org [homepage on the Internet]. c2012. Available from: http://www.theheart.org/article/1339775/ print.do

10. Harder S. Renal profiles of anticoagulants. J Clin Pharmacol. 2011. [Epub ahead of print].

11. Fox KA, Piccini JP, Wojdyla D, et al. Prevention of stroke and systemic embolism with rivaroxaban compared with warfarin in patients with non-valvular atrial fibrillation and moderate renal impairment. Eur Heart J. 2011;32(19):2387-2394.

12. Pisters R, Lane DA, Nieuwlaat R, et al. A novel user-friendly score (HAS-BLED) to assess one-year risk of major bleeding in patients with atrial fibrillation: The Euro Heart Survey. Chest. 2010;138(5):1093-1100.

13. Lip GYH, Frison L, Halperin JL, Lane DA. Comparative validation of a novel risk score for predicting bleeding risk in anticoagulated patients with atrial fibrillation: the HAS-BLED (Hypertension, Abnormal Renal/liver function, Stroke, Bleeding history or Predisposition, Labile INR, Elderly, Drugs and Alcohol Concomitantly) score. J Am Coll Cardiol. 2011;57(2):173-180. 